\title{
URETERAL AVULSION AS A COMPLICATION OF URETEROSCOPY
}

\author{
J.M. ALAPONT, E. BROSETA, F. OLIVER, J.L. PONTONES, F. BORONAT, \\ J.F. JIMÉNEZ-CRUZ
}

Department of Urology, La Fe University Hospital, Valencia, Spain

\begin{abstract}
Purpose: Report and review the literature on ureteral avulsion as a rare complication of ureteroscopy.

Materials and Methods: We analyzed 3 cases of ureteral avulsion in a series of 4,645 ureteroscopic procedures performed from January 1990 to December 2001. We especially report the different managements for this complication.

Results: Due to the different extent of the injury, each patient was treated in a particular way, including a patient managed by means of an endourological approach.

Conclusions: When performing ureteroscopy or using Dormia baskets, one should always bear in mind the possibility of serious complications, including ureteral avulsion or perforation. The use of an extremely careful technique of ureteral insertion, the mandatory placement of a safety guidewire, and a working guidewire, all minimize the risk of untoward events.
\end{abstract}

Key words: ureter; ureteroscopy; lithiasis; intraoperative complications

Int Braz J Urol. 2003; 29: 18-23

\section{INTRODUCTION}

Since its clinical introduction in 1980 by Pérez-Castro \& Martínez-Piñeiro (1), ureteroscopy has experienced an impressive development due to the technical improvements of new and smaller urological armamentarium. Currently, ureteroscopy is a worldwide procedure with varied number of diagnostic and therapeutic possibilities, including treatment of stones, upper urinary tract tumors, strictures, calyceal diverticula, vascular malformations, placement of difficult ureteral stents, and diagnosis of filling defects or hematuria of unknown origin.

However, the technique has complications including bleeding, ureteral perforation, false passage, urinoma, strictures and, in a few cases, ureteral avulsion. We report our experience in the management of ureteral avulsion after ureteroscopy, and we review the few cases published in the literature.

\section{MATERIALS AND METHODS}

Between January 1990 and December 2001 a total of 4,645 transurethral ureteroscopic procedures were performed in our Unit of Endourology. Among those patients 2,508 (53.9\%) procedures were performed under a mild sedative regimen and in outpatient basis. The remainder 2,137 (46.1\%) cases were referred for general anesthesia. Ureteral avulsion occurred in 3 cases as intraoperative complication, and this is the subject of our analysis.

\section{RESULTS}

\section{Case 1}

A 68-year-old man was admitted to the hospital with the main complaint of repetitive left flank pain. The intravenous pyelography (IVP) revealed a 
calculus measuring $5 \times 11 \mathrm{~mm}$ located in the upper third of the left ureter, and a concomitant upper pole renal cyst. After percutaneous cyst puncture, the patient underwent four sessions of extracorporeal shock wave lithotripsy (ESWL) with a Dornier ${ }^{\circledR}$ DL-50 lithotripter that significantly reduced the bulk of the stone but failed to clear the fragments. After a three-week period of watchful waiting, the patient was scheduled for ureteroscopy with a Storz $11.5 \mathrm{~F}$ rigid ureteroscope and laser lithotripsy (Candela ${ }^{\circledR}$ MDL-1 lasertripter). The ureteroscopy was performed under general anesthesia, but the area of stone impaction could not be negotiated because of severe ureteral kinking. Due to the age of the patient, and the long time elapsed from the first procedure, a blind basketing was accepted as a desperate ultimate option to solve the problem in endourological terms. Therefore, a Dormia basket was inserted and after a few withdrawal attempts the stone was eventually dislodged and gently pulled down. However, as the stone was delivered out to the ureteral orifice, a $7 \mathrm{~cm}$ of ureter followed, indicating an ureteral avulsion. It seems likely that the ureter was ruptured at the site of the stone impaction. As a first maneuver and under fluoroscopic guidance, a Teflon guidewire was passed up to the theoretical location of the left renal pelvis. Persistent fever and pain were observed in the following hours, and a computed tomography (CT) scan and an IVP showed both urinary extravasation and a double pigtail stent outside the upper urinary tract. After stabilization with percutaneous nephrostomy and drainage of the urinoma, the patient underwent an ureteroneocistostomy with short mucosal tunnel and Boari flap technique, in order to allow the medial third of the ureter to reach the bladder without any tension. The radiological sequence is shown in Figure-1. Convalescence was uneventful, and an IVP one year after the procedure showed a normal aspect of the collecting system with excellent renal function.

\section{Case 2}

A 64-year-old man presented with a history of continuous right flank pain. An IVP revealed a stone located in the middle third of the right ureter and severe upper urinary tract obstruction with renal function impairment. After 2 failed attempts to place a double pigtail stent, and 2 ESWL procedures without clearance of the stone, the patient was scheduled for ureteroscopy (11.5F) with in situ laser lithotripsy and double pigtail stent placement. After 4,500 pulses of pulsed dye laser (Candela ${ }^{\circledR}$ MDL-1 lasertripter) the stone was not broken, and as direct vision of the impacted stone was possible, a Dormia basket was negotiated behind the calculus and used to retrieve the relatively small stone as gently as possible. The basket was gently pulled down but, as in the aforementioned case, the ureter was avulsed in a length of 5 $\mathrm{cm}$. Due to the previous poor condition of the renal unit, an immediate right nephrectomy was performed. Recovery was satisfactory and serum creatinine remained within the normal range.

\section{Case 3}

A 56-year-old woman was referred to our Unit of Endourology from another hospital due to a failure to treat a right middle ureteral stone after 2 sessions of ESWL. The patient underwent a right ureteroscopy under general anesthesia, and a glide guidewire was advanced up to the collecting system. Afterwards the stone was inadvertently pushed up to the renal pelvis by the irrigating fluid, and an immediate ESWL was consequently decided. The ureteroscope was gently pulled out to the bladder, but a $4 \mathrm{~cm}$ ureteral degloving injury was observed after that maneuver. Because of the limited extent of the ureteral avulsion and the presence of a 3.5 inch safety guidewire, the intussuscepted ureter was advanced again to its original position over a $7 \mathrm{~F}$ double pigtail stent. Following fluoroscopic and sonographic control the catheter was left in place and only a minimal asymptomatic paravesical urinoma was detected. A month later the pushed-up calculus was successfully treated by ESWL. Eight weeks later the double pigtail stent was removed and a subsequent IVP demonstrated minimal delayed pelviocalyceal filling with mild dilation from the upper third of right ureter. The patient remained asymptomatic and was scheduled for ureteroscopy to assess the extent and degree of the residual stricture and further balloon dilation, if required. However, after a three-month follow-up the patient had multiple urinary tract infections, with lack of function of the renal unit and, therefore, a nephrectomy was performed. 


\section{URETERAL AVULSION AS A COMPLICATION OF URETEROSCOPY}
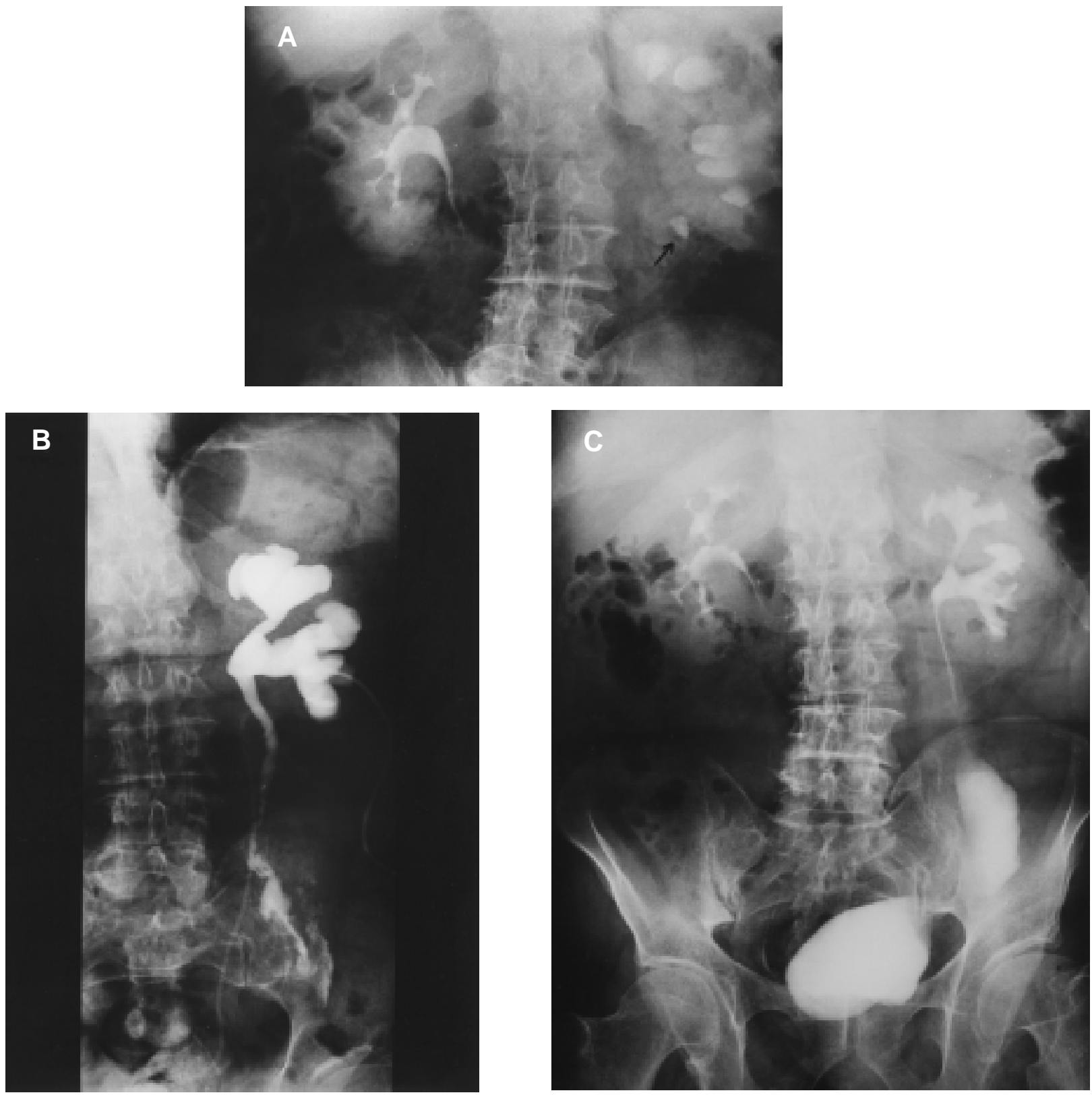

Figure 1- A) Intravenous pyelography (IVP) showing a $5 \times 11 \mathrm{~mm}$ calculus located in the upper third of the left ureter, determining severe upper urinary tract obstruction. B) IVP after ureteroscopy showing urinary extravasation from the middle third of the left ureter. A double pigtail stent is outside of the urinary tract, and a nephrostomy is inserted. C) IVP after Boari flap showing normal function of left kidney.

\section{DISCUSSION}

In the last decade ureteroscopy has become an outstanding breakthrough in the diagnosis and treatment of different ureteral and renal problems. Today it is increasingly used in the management of the common ureteral stones, and such frequent indication has led ureteroscopy to be a worldwide technique, with the expected appearance of multiple types 
of complications (9-20\%), some of them severe, including ureteral perforation or avulsion, bleeding, and urinary tract infection $(2,3)$.

Traditionally, the term ureteral avulsion has been described as an upper urinary tract injury related to the action of blunt trauma, especially from traffic accidents, being the mechanism of injury the result of an acute deceleration/acceleration movement (4). With the advent of endourology, that term is also applied to the extensive degloving injury resulting from a mechanism of stretching of the ureter that eventually breaks at the most weakened site. The first cases were reported by Hart (5) in 1967, and Hodge (6) in 1973, both after difficult manipulation of an ureteral stone with Dormia basket.

Although an infrequent event in the endoscopic management of ureteral calculi $(0.2-1 \%)(7,8)$, with only few cases reported in the literature, ureteral avulsion is a potential serious complication that should always be taken into account when performing such procedures. The incidence in our series was only $0.11 \%$.

Among the potential factors involved in the pathogenesis of ureteral avulsion, the presence of an anomalous ureter, either due to a diseased area or to previous endourologic manipulations, is an important antecedent in the majority of cases. Furthermore, the use of multiple-wire baskets for ureteral stones retrieval have also been implicated, and particularly with regard to the size of the stone (larger than $1 \mathrm{~cm}$ ), and the distance the stone has to cross before exiting through the ureteral meatus (9). Dormia basketing is hardly used in our Unit due to the aforementioned problems. We much prefer the use of grasping forceps to retrieve any fragment after ureteroscopy and laser lithotripsy. Rigid ureterorenoscopes are also a potential source of ureteral avulsion, as shown in the 2 cases reported by Martin et al. (10), and in 1 patient of our own series. In the latter cases the ureteral damage was probably due to the association of a diseased ureter and an improper handling of the endoscopic instrument that eventually caused the degloving injury. During the endoscopic procedure it is very difficult to evaluate, in case of ureteral avulsion, the degree of tissular injury and the extent of layer involvement. In one third of our cases, the theory of an unique mucosal injury seems a sensible hypothesis, because it was very easy to advance the $4 \mathrm{~cm}$ intussuscepted flap to its original position through the safety guidewire previously placed before the attempt of ureteroscope. Whether it was really just a mucosal breakage or a deeper laceration is impossible to ascertain, although the eventual loss of the renal unit would confirm the theory of ureteral avulsion. All injuries occurred during the initial phase of our learning curve. However, with increasing experience we are able to go further in some challenging procedures or difficult cases, and therefore the possibility of ureteral injuries remains a constant threat, including ureteral avulsions, although with the small caliber of our current endoscopes such event seems less probable. Therefore, in the prophylaxis of ureteral avulsion concur especially the endoscopic skill of the urologist, and the adherence to some basic rules, such as using a small ureteroscope, or avoiding Dormia basket retrieval of the stone in cases of large calculus, or partial view of the area where the calculus is impacted.

Diagnosis of ureteral avulsion is most often carried out immediately during the endoscopic procedure, after the recognition of a tubular structure firmly engaged to the ureteroscope following the extraction maneuvers, as it was observed in our 3 patients. However, in some cases a delayed diagnosis is performed in the presence of fever, flank pain, abdominal tenderness, or a flank mass, indicating a retroperitoneal urinoma or abscess secondary to urine leakage (10). Such clinical diagnosis should always be confirmed by ultrasonography, CT scan, IVP, or retrograde pyelography.

Traditionally the treatment of the ureteral avulsion has been a surgical approach, for which the basic aim is to restore the ureteral continuity. Nevertheless, clear guidelines about the best surgical technique are still an unresolved issue. There are some factors that should be taken into account, such as age of the patient, kidney function, level of injury, and length of the ureteral defect. In lower third ureteral lesions, a ureteral reimplantation seems the most rewarding surgical technique, but severe ureteral injuries associated with higher localization or loss of a long segment require several methods of repair, including Boari flap, psoas hitch, transureteroureterostomy, autotransplan- 
tation, or ileal or appendix interposition. The use of a psoas hitch, a Boari flap or a combination of both seems to be the most sensible option, albeit restricted to injuries at or below the pelvic brim. However, Chang \& Koch (11) described a modification of the traditional bladder flap procedure or extended spiral bladder flap for a successful treatment of two patients with upper ureteral injuries. In case of complete avulsion of the ureter at the ureteropelvic junction, a dismembered pyeloplasty is the preferred option. In case of severe tissue loss, autotransplantation, especially in young patients, or ileal interposition, will yield a satisfactory result $(10,12)$. Moreover, an alternative method of successful repair of extensive injuries with appendix interposition was reported in three cases where the conventional techniques were precluded (13-15). In our series, the diverse circumstances of patients and injuries, caused that each case was treated in a different way, including an endourologic approach where the intussuscepted ureter was advanced again to its original position and fixed over a $7 \mathrm{~F}$ double pigtail stent introduced onto a safety guidewire previously placed up to the kidney as a first step in ureteroscopy.

Our experience, and that of others, all suggest that carefully performed ureteroscopy is a superb tool for the urologist, either for diagnostic or therapeutic purposes. However, when performing an ureteroscopy or using Dormia baskets, one should always bear in mind the possibility of serious complications, including ureteral avulsion or perforation. As in many other procedures, a learning curve phenomenon is observed, so we recommend the use of an extremely careful technique of ureteral insertion, and the mandatory placement of a safety guidewire and a working guidewire, to minimize the risks of untoward events.

\section{REFERENCES}

1. Pérez-Castro E, Martínez Piñeiro JA: Ureteral and renal endoscopy. A new approach. Eur Urol. 1982; $8: 117-20$.

2. Schultz A, Kristensen JK, Bilde T, Eldrup J: Ureteroscopy: results and complications. J Urol. 1987; 137:865-6.
3. Blute ML, Segura JW, Patterson DE: Ureteroscopy. J Urol. 1988; 139:510-2.

4. Palmer JM, Drago JR: Ureteral avulsion from nonpenetrating trauma. J Urol. 1981; 125:108-11.

5. Hart JB: Avulsion of the distal ureter with Dormia basket. J Urol. 1967; 97:62-3.

6. Hodge J: Avulsion of a long segment of ureter with Dormia basket. Br J Urol. 1973; 45:328.

7. Puppo P, Ricciotti G, Bozzo W, Introini C: Primary endoscopic treatment of ureteric calculi. Eur Urol. 1999; 36:48-52.

8. Oktay B, Yavasçaoglu I, Simsek Ü, Özyurt M: Intracorporeal pneumatic lithotripsy for ureteral and vesical calculi. Scand J Urol Nephrol. 1997; 31:3336.

9. Abdelsayed M, Onal E, Wax SH: Avulsion of the ureter caused by stone basket manipulation. J Urol. 1977; 118:868-70.

10. Martin X, Ndoye A, Konan PG, Feitosa Tajra LC, Gelet A, Dawara M, et al.: Hazards of lumbar ureteroscopy: apropos of 4 cases of avulsion of the ureter. Prog Urol. 1998; 8:358-62.

11. Chang SS, Koch MO: The use of an extended spiral bladder flap for treatment of upper ureteral loss. J Urol. 1996; 156:1981-3.

12. Shokeir AA: Interposition of ileum in the ureter: a clinical study with long-term follow-up. Br J Urol. 1997; 79:324-7.

13. Juma S, Nickel JC: Appendix interposition of the ureter. J Urol. 1990; 144:130-1.

14. Die Goyanes A, García Villanueva A, Lavalle Echevarría JA, Cabañas Navarro L: Replacement of the left ureter by autograft of the vermiform appendix. Br J Surg. 1983; 70:442-3.

15. Lloyd SN, Kennedy C: Autotransplantation of the vermiform appendix following ureteroscopic damage to the right ureter. Br J Urol. 1989; 63:216-7.

Received: October 10, 2002 Accepted after revision: January 24, 2003

Correspondence address:
Dr. José Miguel Alapont
Service of Urology, La Fe University Hospital
Avda. Campanar 21, 46009
Valencia, Spain
Fax: + 96 386-2600
E-mail: jmalapont@mundofree.com




\section{EDITORIAL COMMENT}

The authors report 3 cases of "ureteral avulsion" following ureteroscopic management, occurring during a ten-year period from 1990-2000. The 3 cases occurred in a series of 4,645 ureteroscopic procedures, representing a rate of $0.11 \%$.

Ureteral avulsion is a rare but well known complication of ureteroscopy, almost always related to the use of an ureteroscope too large to be readily accommodated by the ureter or, in most cases, by an attempt to pull an inadequately fragmented or impacted stone down from the proximal or mid ureter. All of these known risks were evident in the 3 cases reported here. In case one, a $12.5 \mathrm{~F}$ rigid ureteroscope was used, which is no longer a contemporary instrument. In case 2, the size of the ureteroscope was $11.5 \mathrm{~F}$.
As the author state, the stone was not fragmented, and an attempt was made to pull the impacted stone down intact with a basket from the middle third ureter, which is contraindicated. In case 3 , there was a "degloving" injury, which is not a ureteral avulsion. Rather, this is simply an intussusception of ureteral mucosa, which can often be managed with a stent, as the authors did here.

Ureteral avulsion is a rare injury, the risks for which are well described and reiterated in this paper. The best treatment of ureteral avulsion as a complication of ureteroscopy is prevention, not reconstruction, and all of the contraindications to stone management that may lead to that injury are clear in the cases reported here.

Dr. Stevan B. Streem

Head, Section of Stone Disease and Endourology The Cleveland Clinic Foundation Cleveland, Ohio, USA 